\title{
Qualitative and quantitative evaluation of avian demineralized bone matrix in heterotopic beds
}

\begin{abstract}
Objective: To evaluate the osteogenic potential of avian demineralized bone matrix (DBM) in the context of implant geometry. Study Design: Experimental. Animals: Rock pigeons (n四4). Methods: Tubular and chipped forms of DBM were prepared by acid demineralization of long bones from healthy allogeneic donors and implanted bilaterally into the pectoral region of 24 pigeons. After euthanasia at 1, 4, 6, 8, 10, and 12 weeks, explants were evaluated histologically and compared by means of quantitative (bone area) and semi quantitative measures (scores). Results: All explants had new bone at retrieval with the exception of tubular implants at the end of week 1 . The most reactive part in both implants was the interior region between the periosteal and endosteal surfaces followed by the area at the implant-muscle interface. Quantitative measurements demonstrated a significantly (P困 (12) greater percentage of new bone formation induced by tubular implants (80.28困[8.94) compared with chip implants (57.64四虬12). There was minimal inflammation. Conclusions: Avian DBM initiates heterotopic bone formation in allogeneic recipients with low grades of immunogenicity. Implant geometry affects this phenomenon as osteoconduction appeared to augment the magnitude of the effects in larger tubular implants.
\end{abstract}

Keyword: Avian; Demineralized bone matrix; Animal disease 